\title{
Depresión en el adulto mayor, cual es la verdadera situación en nuestro país
}

\author{
Depression in older adults, which is the real situation in our country
}

\section{Sr Editor:}

En los últimos años, el adulto mayor se ha convertido en uno de los principales focos de atención, en virtud del rápido crecimiento de este grupo etario respecto a la población general (1).

Este proceso en los países en desarrollo, a diferencia de los desarrollados, va acompañado de pobreza y falta de políticas de salud; actualmente se estima que sólo $56,3 \%$ de ancianos de las urbes tienen acceso a la seguridad social, encontrándose en situación de pobreza $41,7 \%$ y en pobreza extrema $19,5 \%$ (2).

La Organización Mundial de la Salud afirma que actualmente cinco de las 10 causas más importantes de discapacidad son de origen psiquiátrico y se piensa que para el 2020 la depresión será la segunda causa de discapacidad a nivel mundial (3).

La depresión no es tristeza únicamente, es una enfermedad que debe ser tratada. Contrario a la creencia popular, la depresión no es una parte natural del envejecimiento. La depresión a menudo puede revertirse con tratamiento inmediato y adecuado. Sin embargo, si no se trata, la depresión puede acelerar el deterioro físico, cognitivo y social, dilatar la recuperación de enfermedades y cirugías, provocar un aumento del uso de la atención médica, y el suicidio (4).

A pesar de los esfuerzos realizados en la descripción de la depresión en pacientes geriátricos y en la búsqueda de mejores tratamientos, continúan existiendo consecuencias nefastas tanto para las personas afectadas, como para su entorno familiar y social.

Se han realizado estudios epidemiológicos sobre prevalencia y características de la depresión en poblaciones de adultos mayores de 60 años. La mayoría de ellos revela cierto grado de asociación entre depresión y, por ejemplo, enfermedades crónicas, deterioro cognitivo, grado de instrucción, etc. Sin embargo, siguen siendo necesarios en nuestro país los estudios epidemiológicos sobre depresión en distintas áreas geográficas.

Así mismo, es necesario considerar que la depresión no es una condición inherente al adulto mayor, por lo que su subestimación y un inadecuado abordaje causan un padecimiento innecesario en esta población, y en su entorno.

Es así que se hacen necesarios estudios epidemiológicos poblacionales para poder determinar la prevalencia de esta enfermedad a fin de establecer políticas de salud dirigidas a su prevención. Por este motivo elaboramos un proyecto que tenía como objetivo determinar la prevalencia de depresión en centros geriátricos ya que concentran una gran población de adultos mayores que sería una muestra representativa dado que los adultos mayores de los centros provienen de distintos puntos de la ciudad de Lima y también del interior del país. Se planeaba aplicar un test sencillo y validado, muy utilizado para evaluar depresión, a las personas que voluntariamente aceptaran participar lo cual no afectaría a los ancianos.

El proyecto fue presentado a diversas instituciones que trabajan con adultos mayores, pero no fue aceptada su ejecución, aduciendo diversos motivos diversos como: no aportarle beneficio a las instituciones, antecedentes de malas relaciones con investigadores, molestia de los residentes y que la medición de depresión era constante. ¿Los motivos serán reales o ven las investigaciones de personas externas a las instituciones como un riesgo de difundir el verdadero estado de los adultos mayores en relación a la depresión?, ya que un estudio que revele un alto grado de depresión en la mayoría de los ancianos seria señal que no están haciendo un buen trabajo. 
¿Cuál será la situación de los adultos mayores residentes en los centros geriátricos? es una duda que debe ser resuelta y una duda mucho más grande es la situación de los adultos mayores de todo el país, de aquellos que no cuentan con servicios de salud o familiares que muestren interés en ellos.

Mauro Jonis

Instituto de Investigación en Ciencias Biomédicas, Universidad Ricardo Palma. Lima, Perú.

Diana Llacta

Instituto de Investigación en Ciencias Biomédicas, Universidad Ricardo Palma. Lima, Perú.

\section{REFERENCIAS BIBLIOGRÁFICAS}

1. Instituto Nacional de Estadística. Perfil socio- demográfico de la tercera edad. Lima: INEI; 1995.

2. Mayta P, Zelada M. ¿Estamos preparados para la atención primaria del adulto mayor? Rev Perú Med Exp Salud Pública. 2006; 23(1):67-69.

3. De la Torre J, Shimabukuro R, Varela L, et al. Validación de la versión reducida de la escala de depresión geriátrica en el consultorio externo de geriatría del Hospital Nacional Cayetano Heredia. Acta Med Per. 2006; 23(3): 144-147.

4. Kurlowics L, Greenberg S. La escala de depresión geriátrica (GDS, por sus siglas en inglés). Try this: Best practices in nursing Care to older adults (Internet). 2007 (Citado el 17 de mayo de 2010); Disponible en: http://consultgerirn.org/uploads/ File/trythis/try_this_4_span.pdf. 